\title{
Coercive treatment and stigma: is there a link? Wolfgang Gaebel
}

\author{
Address: Department of Psychiatry and Psychotherapy, Heinrich-Heine-University Düsseldorf, Rhineland State Clinics Düsseldorf, Bergische \\ Landstraße 2, 40629 Düsseldorf, Germany \\ from WPA Thematic Conference. Coercive Treatment in Psychiatry: A Comprehensive Review \\ Dresden, Germany. 6-8 June 2007 \\ Published: 19 December 2007 \\ BMC Psychiatry 2007, 7(Suppl I):S2 doi:10.1 I86/I47I-244X-7-SI-S2
}

This abstract is available from: http://www.biomedcentral.com/I47I-244X/7/SI/S2

(C) 2007 Gaebel; licensee BioMed Central Ltd.

Coercive treatment comprises compulsory admission of patients as well as coercive treatment measures. Both are sometimes inevitable elements of psychiatric care, with the aim to prevent danger from patients and other persons. The stigma of mental illness is still one of the most challenging treatment obstacles. It is not limited to patients and their families, but is also attached to treatment methods and institutions. The link between coercive treatment and stigma of mental illness is complex. The fact that compulsory treatment is administered within the treatment of mentally ill persons, but not of patients with somatic illness, shapes the public's impression that mentally ill persons are different from others and potentially unpredictable and dangerous - with fear and social distance as consequences. These negative attitudes are also strengthened in sensational media reports on patients being involuntarily admitted to psychiatric institutions, which thereby also become associated with a negative image. It is not yet clear, whether and how mental health professionals' attitudes influence their practice of coercive treatment. In Germany, coercive treatment underlies strict legal regulations. Nevertheless, occasions and justifications for coercive treatment in clinical practice may vary due to individual tolerance limits and competence in deescalation techniques which in turn are influenced by beliefs, attitudes and professional experiences of the ward staff. In case of an interaction between stigma and coercive treatment, the question arises, whether the frequency of compulsory admissions and coercive treatment measures can be reduced by educational and stigma-oriented interventions. Empirical evidence will be discussed examining the link between coercive treatment and stigma, focused on public and professionals' attitudes and behavior as well as patients' perspectives. 